\title{
Vortex interaction in patches of randomly placed emergent cylinders
}

\author{
Ana M. Ricardo \\ Instituto Superior Técnico, ULisboa, Portugal \\ Laboratory of Hydraulic Constructions, EPFLausanne, Switzerland \\ Simona Di Carlo \\ Universit degli Studi di Cassino e del Lazio Meridionale, Italy \\ Mário J. Franca \& Anton J. Schleiss \\ Laboratory of Hydraulic Constructions, EPFLausanne, Switzerland \\ Pedro M. Sanches \& Rui M.L. Ferreira \\ Instituto Superior Técnico, ULisboa, Portugal
}

\begin{abstract}
The flow field of multiple-cylinder configurations exhibits complex interactions between shear layers, vortexes and wakes. For high stem-Reynolds numbers, the flow is turbulent and, low and intermediate areal number-densities of cylinders, and turbulence is produced mostly by the work of Reynolds shear stresses in the horizontal plane ( $u v$ component) against the time-averaged shear rate characteristic of vertical-axis vortex shedding in the wake of cylinders. The spatial pattern of turbulent production and of other terms of the equation of conservation of Turbulent Kinetic Energy (TKE) is thus mostly determined by the interaction of vortexes shed by individual cylinders and by the distance between cylinders. The main objective of this paper is to advance on the understanding of vortex interaction in patches of randomly placed emergent and rigid cylinders. In particular, the relation between cylinder Strouhal numbers, vortex decay and vortex path statistics is investigated for isolated cylinder and for a cylinder within an array of randomly placed cylinders with a areal-number density of 980 cylinders $/ \mathrm{m}^{2}$. Results are compared to shed light on the influence of neighbouring cylinders. An experimental database acquired with 2D Particle Image Velocimetry (PIV) was explored. A methodology to detect vortexes in 2D flow fields is proposed. It features a point-based criterion and a global search to detect all the possible vortex core locations, combined with a curve-based criterion, to decide whether the detected point corresponds to a vortex, depending on the geometry of streamlines. The results show a decrease on the amount of vortexes and a shorter vortex life for the cylinder within the array when compared with the isolated cylinder. The averaged vortex path is also affected by the presence of neighbouring cylinders. Concerning the Strouhal number, the normalized shedding frequency is approximately the same for both studied cases.
\end{abstract}

\section{INTRODUCTION}

Flow around circular cylinders is a classical problem of fluid mechanics due to its common occurrence in many applications. The flow around an isolated cylinder has been extensively studied, (comprehensive reviews in Williamson 1996, Zdravkovich 1997, Zdravkovich 2003) and Sumner (2010) presented a review of studies about the effects of a second cylinder placed in close proximity, arranged in tandem, side-by-side, or in staggered configurations. Less well studied are the changes to the flow when a cylinder is placed in an array of cylinders, specially when they are randomly placed. The flow field of multiple-cylinder configurations involve complex interactions between wakes, von Kármán vortex streets and shear layers (Sumner 2010).
For high stem-Reynolds numbers, defined as $R e_{p}=U d / v$, where $U$ is the time- and space-averaged velocity in the vicinity of the cylinder, $d$ is the cylinder diameter and $v$ is the kinematic viscosity of the fluid, the flow is turbulent. It has been shown (Ferreira et al. 2013, Ricardo 2013) that, for low and intermediate areal number-densities of cylinders, turbulence is produced mostly by the work of Reynolds shear stresses in the horizontal plane ( $u v$ component) against the time-averaged shear rate characteristic of vertical-axis vortex shedding in the wake of cylinders. The spatial pattern of turbulent production and of other terms of the equation of conservation of Turbulent Kinetic Energy (TKE) is thus mostly determined by the interaction of vortexes shed by individual cylinders and by the distance between cylinders. 
The main goal of this paper is to advance the understanding of vortex interaction in patches of randomly placed emergent and rigid cylinders, exploring two experimental databases acquired with 2D Particle Image Velocimetry (PIV) system. One of the databases includes instantaneous velocity maps downstream of a cylinder within an array of cylinders randomly placed. The second database consists in the instantaneous flow field downstream of an isolated cylinder.

Vortexes are among the most important and coherent structures that control the dynamics of flow fields (Banks \& Singer 1994) and their study constitutes an important research topic, from both theoretical and practical points of view. The concept of vortex is loosely expressed as a helical pattern of flow in a localized region. However, no formal mathematical definition for vortex exists, for instance employing concepts of vorticity or helicity (Banks \& Singer 1994). An operative definition, provided by Robinson (1991), is that a vortex exists when instantaneous streamlines mapped onto a plane normal to the vortex core exhibit a roughly circular or spiral pattern, when viewed from a reference frame moving with the center of the vortex core. The drawback associated with this definition is that it requires the ability to recognize and track a vortex core.

Visualization of vortexes plays an important role in the understanding of the swirling features in flow fields. A literature review shows that several criteria have been developed for vortex detection, but the essential characteristics are hard to capture, and none of the existing criteria is entirely satisfactory (Banks and Singer 1994, Sadarjoen et al. 1998, Jiang et al. 2005). The main research efforts have been placed in the visualisation of vortexes in complex 3D flows. According to Sadarjoen et al. (1998), the criteria in vortex detection can be classified in two groups: point- and curve-based criteria. The former includes methodologies in which a local quantity is sampled or calculated at a given point in a flow field while the latter makes use of local geometric properties of streamlines. The point-based criteria reveal vortical structures with varying degrees of success, it has been reported that it fails for some cases (Sadarjoen et al. 1998). On the other hand, curve-based criteria seems to detect vortexes more successfully, but it is computationally more expensive.

The present work proposes a methodology to detect vortexes, restricted to two-dimensional flow fields, combining a point-based criterion, embedded in a global search to detect all the possible vortex core locations, with a curve-based criterion to decide whether the detected point corresponds to a vortex, depending on the geometry of streamlines.

Concerning the organization of this article, after this introductory section it follows the description of the laboratory facilities and the two experimental tests carried out. Then, in section 3, the methodology applied for vortex detection is presented and it is followed by the results. The article finishes with the main conclusions.

\section{EXPERIMENTAL FACILITIES}

The experimental work was carried out in a $12.5 \mathrm{~m}$ long and $0.408 \mathrm{~m}$ wide tilting recirculation flume of the Laboratory of Hydraulics of IST. The flume has glass side walls, enabling flow visualization and laser measurements (Fig. 1). The flume bottom was covered with a horizontal layer of gravel and sand. The flow was controlled by a venetian-blind gate at the flume's outlet.

Two experimental tests were considered in the present work:

- Test A-Array of cylinders: Cylinders with $1.1 \mathrm{~cm}$ of diameter were randomly placed with a uniform distribution of 980 cylinders $/ \mathrm{m}^{2}$ along a $3.5 \mathrm{~m}$ long reach. This reach populated with vertical cylinders had two gaps to allow velocity measurements. The width of the measuring gaps is equal to the mean inter-stem distance $(0.032 \mathrm{~m})$. The data presented herein was acquired in the upstream measuring gap, which is located about $2 \mathrm{~m}$ after the begin of the array of cylinders. Figure 2 shows a plan view of part of the reach covered by cylinders. Within the array the flow was an uniform regime.

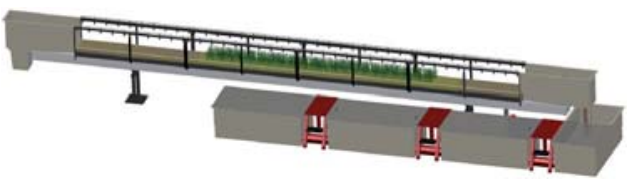

Figure 1. Sketch of the flume where the experiments were performed.

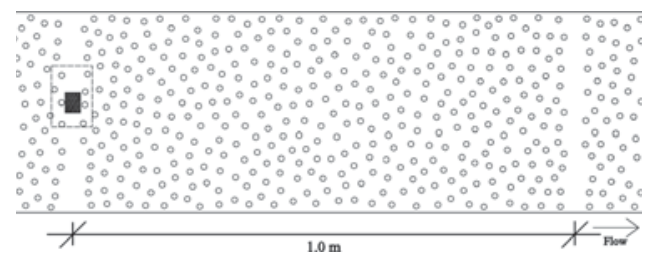

Figure 2. Plan view of part of the reach covered by cylinders. The dashed rectangle points out the region where the velocity maps used herein were acquired. The filled rectangle represents the area where the vortex search was performed. The arrow indicates the flow direction. 
- Test B-Isolated cylinder: An isolated cylinder, also with diameter of $1.1 \mathrm{~cm}$, was placed upstream of the reach populated with cylinders of test A. The flow upstream of cylinders' array was gradually varied decelerating towards downstream. Figure 3 presents the longitudinal profile of the free surface on the region where the isolated cylinder was placed.

The experimental data acquisition consisted in a instantaneous velocity maps, in the horizontal plane, obtained with a $532 \mathrm{~nm}, 30 \mathrm{~mJ}$ 2D Particle Image Velocimetry (PIV) system. The PIV is an optical technology that allows obtaining the fluid velocity by measuring seeding particles velocity. The system is composed of the laser head, the power supply, the CCD camera and the acquisition system. It is based on a double-cavity laser which allows the user to set the delay between two laser pulses. During experimental tests presented herein, the PIV was operated at a sampling rate of $15 \mathrm{~Hz}$ and with a time between pulses of $1500 \mu \mathrm{s}$. The seeding particles used in this experimental work for flow visualization were made of polyamide, with specific gravity of 1.03 and diameter range of 30 to $70 \mu \mathrm{m}$, with an averaged diameter of $50 \mu \mathrm{m}$.

The horizontal maps were acquired at $3.8 \mathrm{~cm}$ above the bed, which is, for test $\mathrm{A}$, in the region where the flow is mainly controlled by the vertical cylinders. For each test, 4500 images couples were acquired, corresponding to 5 minutes of consecutive data. The CCD of the camera used has a size of $1600 \times 1200 \mathrm{px}^{2}$ and the image correlation was performed with interrogation areas of $16 \times 16 \mathrm{px}^{2}$ with $50 \%$ of overlap. An area of roughly $12 \times 9 \mathrm{~cm}^{2}$ was measured, yielding to interrogation volumes of $0.6 \times 0.6 \times 2 \mathrm{~mm}^{3}$, since the laser light sheet has approximately $2 \mathrm{~mm}$ of thickness.

The theory of Hjemfelt \& Mockros (1996) reveals that the seeding used allows the identification of turbulent structures with the frequencies lower than $40 \mathrm{~Hz}$ with a significance level of 0.95 . Since the PIV was operated at $15 \mathrm{~Hz}$, the Nyquist frequency is equal to $7.5 \mathrm{~Hz}$. Therefore, it can be concluded that the seeding particles used ensure the quality of the data acquired in the time

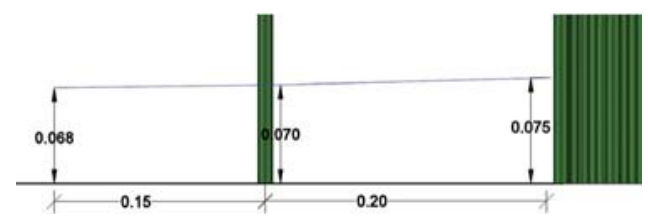

Figure 3. Lateral view of the reach where the single cylinder was placed. The blue line represents the freesurface. The arrow indicates the flow direction. The dimensions are in SI units (m)
Table 1. Flow properties.

\begin{tabular}{lllll}
\hline & $h(\mathrm{~m})$ & $U(\mathrm{~m} / \mathrm{s})$ & $T\left({ }^{\circ} \mathrm{C}\right)$ & $R e_{p}(-)$ \\
\hline Test A & 0.084 & 0.131 & 24 & 1584 \\
Test B & 0.070 & 0.158 & 24 & 1910 \\
\hline
\end{tabular}

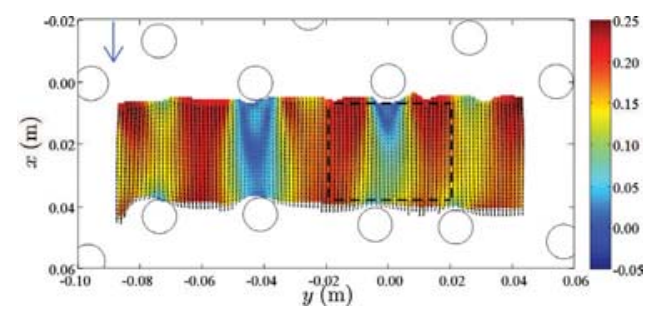

Figure 4. Time-averaged velocity map for test A. The arrow indicates the flow direction. The dashed rectangle delimits the region where the vortexes were searched.

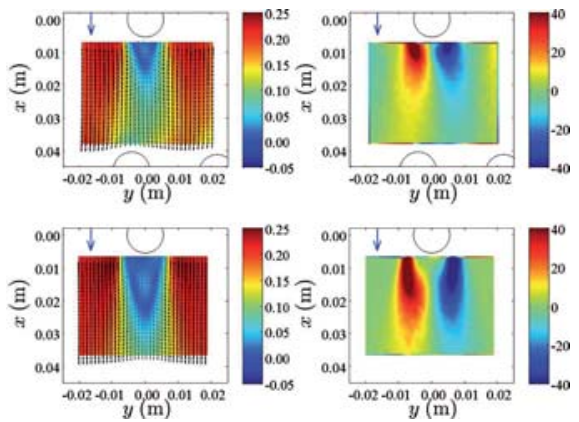

Figure 5. Time-averaged velocity (left) and vorticity (right) maps for test A (top) and test B (bottom). The arrow indicates the flow direction.

domain. In the space domain, applying Taylor's frozen turbulence hypothesis (Tennekes \& Lumley 1972 , p. 253) and considering a mean velocity of $0.13 \mathrm{~m} / \mathrm{s}$, the frequency $f_{c}=40 \mathrm{~Hz}$ corresponds to a turbulent length scale $\lambda_{c}=3.3 \mathrm{~mm}$. This means that the velocity of eddies smaller than $3.3 \mathrm{~mm}$ may be measured with less than $95 \%$ confidence employing this seeding particles.

The tests were performed with a discharge of $4.51 \mathrm{~s}^{-1}$. Table 1 presents flow properties for each test, where $h$ is the flow depth, $U$ is the mean (timeand space-averaged) flow velocity, $T$ is the water temperature during the experiments, and $R e_{p}$ is the stem Reynolds number. The diameter of the cylinders is $d=0.011 \mathrm{~m}$ and $v$ depends on the water temperature.

Figure 4 presents the time-averaged velocity map of test A, showing the heterogeneity of the 
flow, in the space inter-cylinders, with zones of low velocity at the cylinder's wake alternated by high velocity zones between cylinders. The vortexes were searched in a rectangular area, centred downstream of a cylinder, with dimensions $4 \mathrm{~d} \times 3 \mathrm{~d}$ (width $\times$ length) in both tests. Time-averaged velocity and vorticity maps for tests $\mathrm{A}$ and $\mathrm{B}$ are presented in Figure 5.

\section{METHODOLOGY}

The methodology proposed in the present work for vortex detection comprises two main steps.

- First, for a given instantaneous velocity map, a global search is performed to identify possible vortex cores, finding the points where both longitudinal $(u)$ and lateral $(v)$ velocity components are zero. Figure 6 exemplifies an instantaneous velocity map in test A, represented by a vector plot, overlapped by the isolines of $u=0$ (green lines) and $v=0$ (red lines). The black points, marking the intersection of the two isolines, correspond to the location of the cores of the possible vortexes for the actual instantaneous velocity field.

- After the identification of all possible vortexes in a given region, a more local approach based on the streamlines neighbouring each point are analysed to distinguish between vortex cores or stagnation points. For each point, four streamlines are drawn around starting at $0, \pi / 2, \pi$ and $3 \pi / 2$ in the circle, with radius smaller than the size of interrogation areas, centred in that point. If at least two streamlines are closed (making a $2 \pi$ revolution) and bounded and if there is a circulation pattern in the near vicinity of the point, then candidate is considered a vortex.

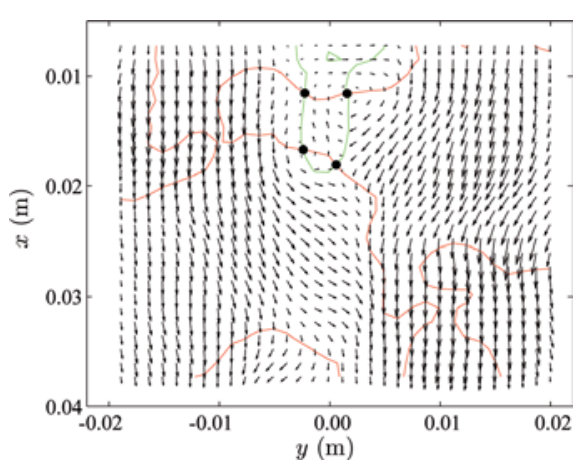

Figure 6. Isolines of zero longitudinal (green) and lateral (red) velocity. The vector plot represents an instantaneous velocity map filtered with a Gaussian filter.
The streamlines for the cross points identified in Figure 6 are represented in Figure 7, where two vortexes were found (the two sub-figures on the right hand side).

A database of vortexes is built, each vortex marked by the number of the vortex map to which it belongs and by a timestamp and identified by the coordinates of the core and the value of the nearcore circulation (including signal). This database is then processed to track vortexes into vortex paths. The key steps are summarized below.

1. The first vector map with vortexes is used to initialize a record of open paths; the initial points of these paths are the coordinates of the vortexes in that map.

2. The next vector map with vortexes is processed. The open paths for which the difference between the timestamp of the current vector map and timestamp of their last entry is larger than a given flow-dependent threshold are closed (and killed if there was only one entry). The remaining open paths may be continued by the vortexes with the timestamp of the current vector map.

3 . To determine if the vortexes with a given timestamp continue existing open paths or constitute initial points of new paths, four situations are considered: a) the current vector map has one vortex and there is only one open path; b) the current vector map has only one vortex but there are more than one open paths; c) the current vector map has more than one vortex and there is only one open path; d) the current vector map has more than one vortex and there are more than one open paths. The general criteria to map vortexes to paths employs a correlation analysis, with the key restriction that vortexes are not allowed to travel upstream.

4. Once step 3 is finished, the record of open paths is updated and the process returns to step 2 .
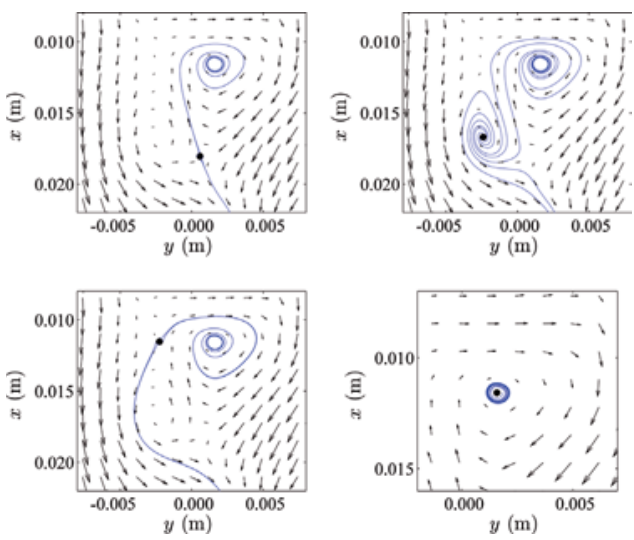

Figure 7. Streamlines for each point of zero velocity. 
Databases of positive and negative vortex paths are thus built. Statistical treatment of these databases allows for the determination of mean vortex velocity, mean angle of vortex motion, path length distribution and vortex shedding frequency, among others.

\section{RESULTS}

The methodology described in the previous section was applied to the sequence of velocity maps whose time-average spatial velocity distribution is shown in Figure 5, distinguishing vortexes with positive and negative circulation.

Table 2 summarizes statistics of the detected vortexes. The first two columns, labelled as " $T$ " and "C", identify the test and the sign of the vortex circulation, respectively. The sign of circulation was defined by the right-hand rule, therefore a vortex with counter-clockwise circulation has a positive circulation, and it is mentioned herein as a positive vortex for simplification. In Table $2, N$ stands for the number of vortexes detected, $\mu$ is the expected value of the longitudinal component of the path length and $\sigma$ is the respective standard deviation. The last three columns in the same table indicates the percentage of vortex that were detected between the shedding cylinder and $1 d, 2 d$ and $3 d$ further downstream.

The amount of detected vortexes in the test with the isolated cylinder is significantly larger in the array of cylinders. It is also observed that in test $\mathrm{B}$ the vortex live longer than in test $\mathrm{A}$, in the sense that more vortexes are found further downstream in the former. A visual representation of those observations is presented in Figures 8 and 9, where the location of each detected vortex is represented, the blue points being the negative vortexes and the red points being the positive vortexes.

As expected due to the known anti-symmetric pattern of vorticity on the wake of a cylinder, the number of positive and negative vortexes detected in test $B$ is similar. In test A, the amount of positive vortexes is $7 \%$ higher than the negative ones, which

Table 2. Elementary statistics of detected vortexes and paths for each test.

\begin{tabular}{|c|c|c|c|c|c|c|c|}
\hline \multirow[b]{2}{*}{$T$} & \multirow[b]{2}{*}{ C } & \multirow[b]{2}{*}{$N$} & \multirow[b]{2}{*}{$\mu(\mathrm{m})$} & \multirow[b]{2}{*}{$\sigma(\mathrm{m})$} & \multicolumn{3}{|c|}{$\%$ Vortexes in } \\
\hline & & & & & $1 d$ & $2 d$ & $3 d$ \\
\hline \multirow[t]{2}{*}{ A } & + & 1698 & 0.0063 & 0.0046 & 80.3 & 17.7 & 2.1 \\
\hline & - & 1577 & 0.0059 & 0.0046 & 83.3 & 14.9 & 1.8 \\
\hline \multirow[t]{2}{*}{ B } & + & 3317 & 0.0087 & 0.0058 & 52.3 & 38.5 & 9.2 \\
\hline & - & 3390 & 0.0081 & 0.0057 & 56.6 & 34.7 & 8.6 \\
\hline
\end{tabular}

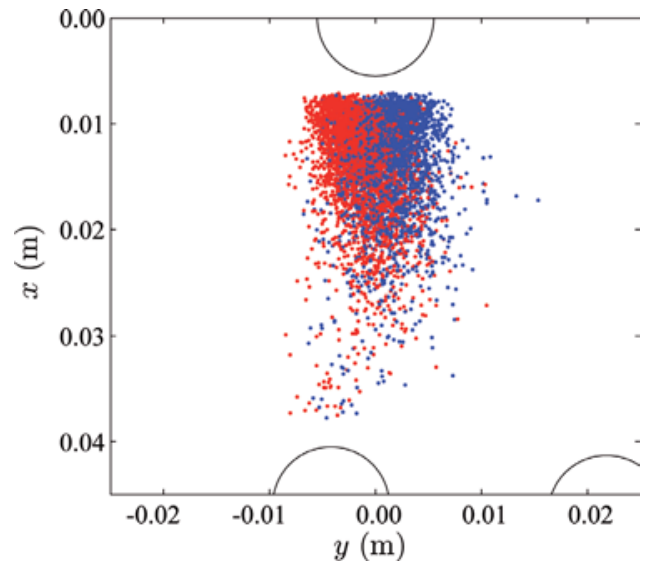

Figure 8. Location of the vortex cores for test A.

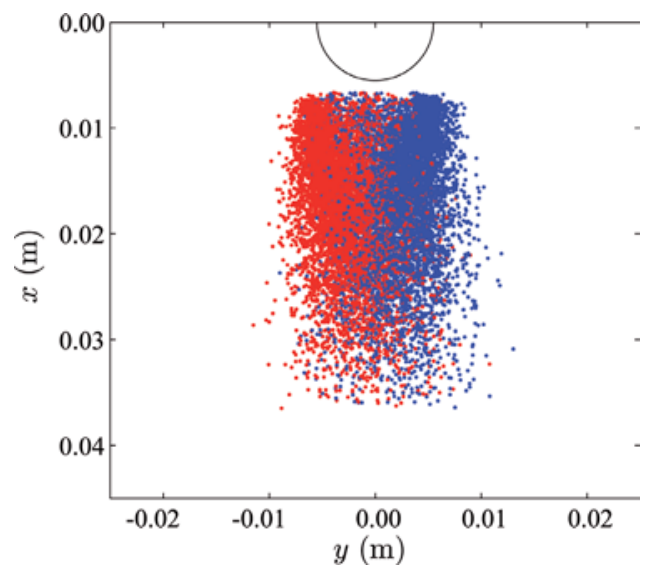

Figure 9. Location of the vortex cores for test B

may express the influence of spatial asymmetries in the approaching flow. The distribution of vortexes along streamwise direction is presented, for both experimental tests, in Figures 10 and 11 for positive and negative vortexes, respectively.

Comparing the distributions for test A with those for test $\mathrm{B}$, one concludes that in the former the vortexes are mainly concentrated close to the shedding cylinder while in the case of the isolated cylinder, vortexes subsist at larger distances downstream. This behaviour is common to positive and negative vortexes.

To get more information about how the vortexes travel downstream and to obtain its shedding frequency, vortex paths were determined. Paths of the positive and negative vortexes, for test $\mathrm{A}$ and $\mathrm{B}$, are shown in Figure 12. 

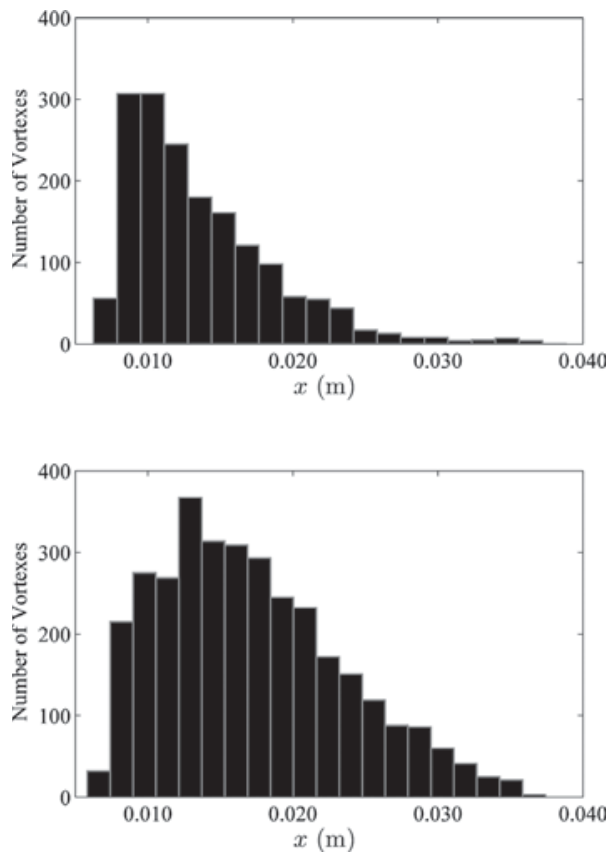

Figure 10. Distribution of the positive vortexes along streamwise direction in the array of cylinders (top) and for the isolated cylinder (bottom).
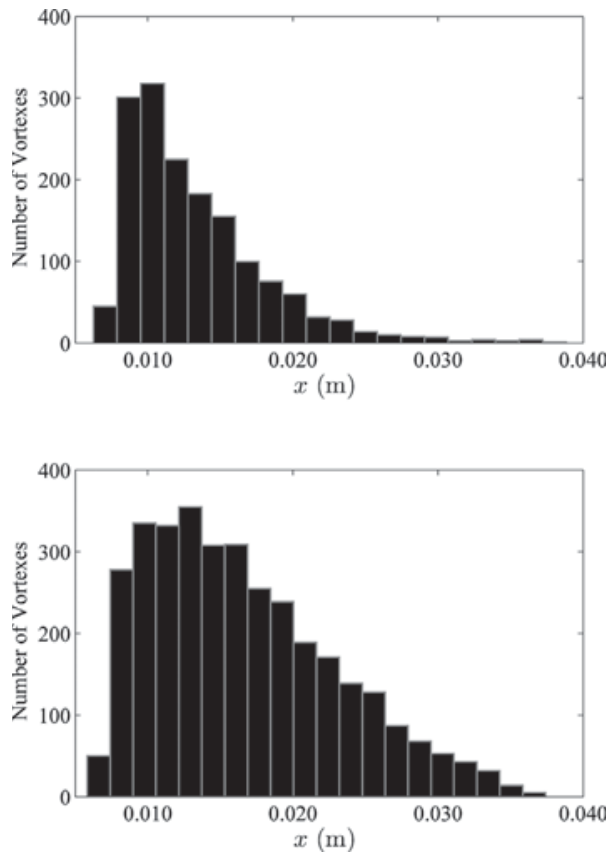

Figure 11. Distribution of the negative vortexes along streamwise direction in the array of cylinders (top) and for the isolated cylinder (bottom).

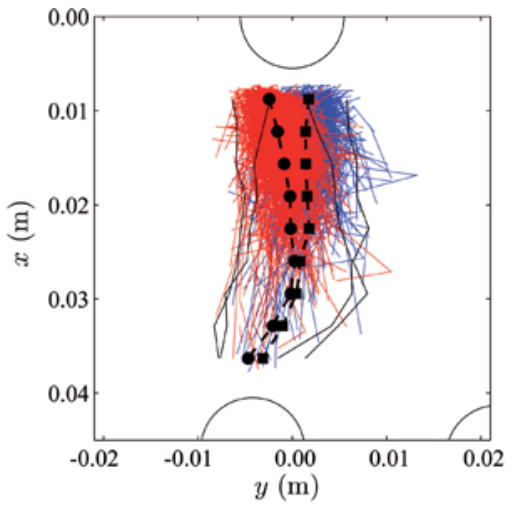

(a)

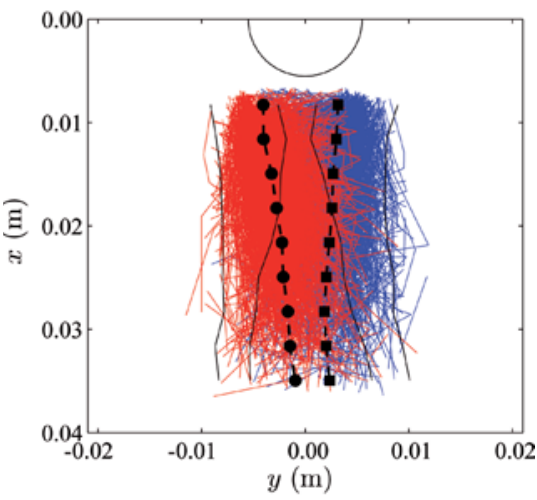

(b)

Figure 12. Detected paths for positive (red lines) and negative (blue lines) vortexes for a) test $A$ and $b$ ) test $B$. The black dashed lines are the averaged paths, with circle markers to positive vortexes and square markers to negative vortexes.

The vortexes shed by an isolated cylinder (test B) travel downstream following an approximately linear path. Negative and positive vortex paths seem to converge until stabilizing into parallel paths $0.04 d$ apart. In test $\mathrm{A}$, the vortex paths shows a curvature when arriving close to the nearest downstream cylinder, being effectively drawn to it. This curvature may be an effect of the pressure field gradient in front of that cylinder.

Figure 13 shows the distribution of vortex shedding periods for both tests. One can observe that the expected value of the period is slightly smaller in test B, which may be due to the higher mean flow velocity. Test A shows a more concentrated period distribution around the mode, expressed in a smaller value of the standard deviation. 

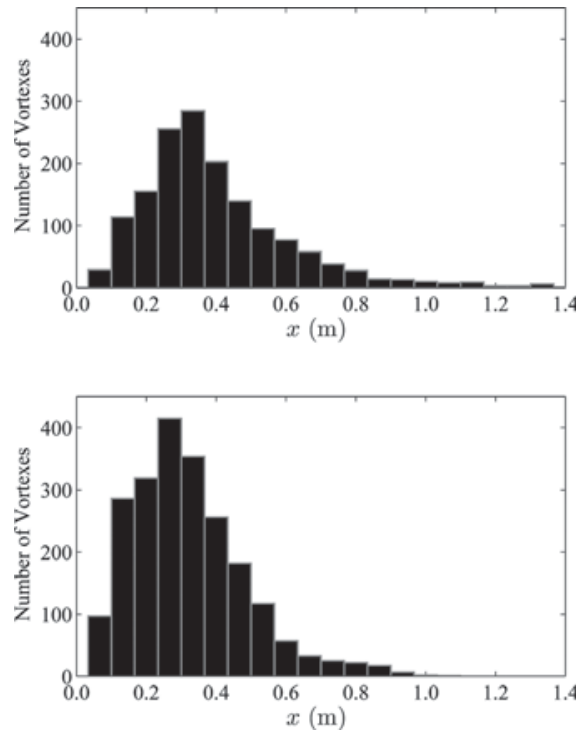

Figure 13. Distribution of vortex periods for test $\mathrm{A}$ (top) and test B (bottom).

The mean period of the presented distributions are $0.40 \mathrm{~s}$ and $0.32 \mathrm{~s}$, for test $\mathrm{A}$ and $\mathrm{B}$, respectively, what corresponds to mean shedding frequencies, $f$, of $2.47 \mathrm{~Hz}$ and $3.11 \mathrm{~Hz}$. Thus, the Strouhal number, a normalized frequency defined by $S t=f d / U$, is 0.208 in test A and 0.216 in test B.

\section{DISCUSSION AND CONCLUSIONS}

The present work introduces a methodology to detect vortexes combining a global and a local procedure, based in detection of zero velocity points and analysing streamlines. This methodology was applied to provide evidence - vortex spatial distribution and vortex paths - to study the interaction of vortex streets within arrays of cylinders, relatively to the reference case of an isolated cylinder.

The interaction of vortex streets may be expressed by the spatial distribution of locations of vortex cores and by the length of its path, relatively to the case of a single cylinder. It was observed that more vortexes are found in the case of an isolated cylinder and those vortexes can travel longer downstream before disappearing.

The path of the vortexes is also affected by the presence of other cylinders. In the case of an isolated cylinder the positive and negative vortexes travel downstream following, initially, linear converging paths, and latter, linear parallel paths. Within an array of cylinders the vortexes are deviated from a straight path presumably due the pressure field of the cylinders downstream.
In what concerns the Strouhal number, the presence of neighbouring cylinders does not impact the results obtained for the isolated cylinder. In both test Strouhal number was $\approx 0.21$, the well known Strouhal number characterizing the vortex sheddingin an isolated cylinder for $R e \sim 10^{3}$.

Future developments depend on the enlargement of the database of vortex paths with other cylinders, subjected to different arrangements of neighbours. This will allow for a clarification of the role of upstream and downstream cylinders in the longitudinal survival rate of vortexes and on Strouhal number of a given cylinder.

\section{ACKNOWLEDGMENTS}

This research funded by the Portuguese Foundation for Science and Technology (FCT) through the project PTDC/ECM/117660/2010.

\section{REFERENCES}

Banks, D.C. \& B.A. Singer (1994). Vortex tubes in turbulent flows: identification, representation, reconstruction. In Proceedings of the conference on Visualization' 94, pp. 132-139. IEEE Computer Society Press.

Ferreira, R.M., A.M. Ricardo, \& K. Koll (2013). Experimental characterization of the tke budget in flows with emergent vegetation. In Proceedings of 35th IAHRWorld Congress, pp. 1-10. IAHR.

Hjemfelt, A.T. \& L.F. Mockros (1996). Motion of discrete particles in a turbulent fluid. Applied Science Research 16, 149-161

Jiang, M., R. Machiraju, \& D. Thompson (2005). Detection and visualization of vortices. In The Visualization Handbook, pp. 295. Academic Press.

Ricardo, A.M. (2013). Hydrodynamics of turbulent flows within arrays of circular cylinders. In Proceedings of 35th IAHR World Congress, pp. 1-12. IAHR

Robinson, S.K. (1991). Coherent motions in the turbulent boundary layer. Annual Review of Fluid Mechanics 23(1), 601-639.

Sadarjoen, I., F. Post, B. Ma, D. Banks, \& H.-G. Pagendarm (1998, Oct). Selective visualization of vortices in hydrodynamic flows. In Visualization '98. Proceedings, pp. 419-422.

Sumner, D. (2010). Two circular cylinders in cross-flow: A review. Journal of Fluids and Structures 26(6), 849-899.

Tennekes, H. \& J. Lumley (1972). A first course in turbulence. The MIT press.

Williamson, C.H. (1996). Vortex dynamics in the cylinder wake. Annual Review of Fluid Mechanics 28(1), 477-539.

Zdravkovich, M.M. (1997). Flow around Circular Cylinders: Volume 1: Fundamentals, Volume 2. Oxford University Press.

Zdravkovich, M.M. (2003). Flow around Circular Cylinders: Volume 2: Applications, Volume 2. Oxford University Press. 